\title{
Nonlinear Rolling Motion of Ship in Random Beam Seas
}

Jia-Yang Gu

School of Naval Architecture and Ocean Engineering, Jiangsu University of Science and Technology, Zhenjiang, Jiangsu 212003, Peoples Republic of China., gujiayang@yahoo.com.cn

Follow this and additional works at: https://jmstt.ntou.edu.tw/journal

Part of the Engineering Commons

\section{Recommended Citation}

Gu, Jia-Yang (2004) "Nonlinear Rolling Motion of Ship in Random Beam Seas," Journal of Marine Science and Technology. Vol. 12: Iss. 4, Article 6.

DOI: $10.51400 / 2709-6998.2247$

Available at: https://jmstt.ntou.edu.tw/journal/vol12/iss4/6

This Research Article is brought to you for free and open access by Journal of Marine Science and Technology. It has been accepted for inclusion in Journal of Marine Science and Technology by an authorized editor of Journal of Marine Science and Technology. 


\title{
NONLINEAR ROLLING MOTION OF SHIP IN RANDOM BEAM SEAS
}

\author{
Jia-Yang $\mathrm{Gu}^{*}$
}

Key words: nonlinear roll, melnikov function, phase space flux, ship capsize, safe basin, unsafe area, heteroclinic orbit.

\begin{abstract}
The highly nonlinear roll and capsizing motion of ships in random seas are analyzed in this paper by utilizing the Melnikov function and phase space flux. Influence factors on the phase space flux, such as significant wave height, significant wave frequency, nonlinear righting arm and damping characteristic, have taken into consideration. As an example, when a fishing vessel of $30.7 \mathrm{~m}$ long and $6.9 \mathrm{~m}$ wide is considered to sail in the seas of ISSC wave spectrum, the Melnikov function in time domain is computed. Also, the relation between the phase space flux and the Melnikov function has attained and the influence of significant wave height on the phase space flex has discussed. It is shown that the phase space flux is monotonically increasing as the significant wave height increases, while the safe basin is decreasing rapidly.
\end{abstract}

\section{INTRODUCTION}

A ship sailing in sea can be considered as an autonomous system exposed to many hazardous circumstances. Among which capsizing is the most catastrophic one since it can result in the heavy losses of ship and human lives. Thus, national and international rules have protocoled to regulate the stability criteria for ships. Based on long-time experience, the characteristics and area under the restoring moment curve have been prescribed. In this way, it intends to ensure that the potential energy due to restoring moment in calm sea has to be larger than the work done by a possible heeling moment. Unfortunately, the existing stability criteria are not satisfactory, owing to the facts that may ships have ever capsized. Therefore, few researchers envisaged to reconsider the ship stability criteria by utilizing the methodology from the state-ofthe-art in nonlinear dynamics.

Paper Submitted 04/24/04, Accepted 09/09/04. Author for Correspondence: Jia-Yang Gu. E-mail: gujiayang@yahoo.com.cn.

*School of Naval Architecture and Ocean Engineering, Jiangsu University of Science and Technology, Zhenjiang, Jiangsu 212003, Peoples Republic of China.
The interest in nonlinear ship dynamics started to surge in the 1970s. While at that time the computers were still at an embryonic stage and it was perhaps inevitable that the prominence to the nonlinear ship dynamics would be given to analytical methods. Unfortunately, these methods are not only extremely laborious but also requiring that the nonlinearities be weak, thus leading to eliminate much of the most dynamically interesting part of the behavior.

The vast increase in computer power realized in the 1980s and 1990s brought a wide range of new techniques of numerical analysis of nonlinear dynamical systems into the limelight. During these years the study of nonlinear rolling in beam seas continued to be topical. Nayfeh and Khdeir [6, 7], Papanikolaou and Zaraphonitis [9] presented studies of large amplitude rolling based on a combination of analytical perturbation based techniques and digital analogue simulations. Virgin [19] concentrated on the onset of chaotic roll oscillations occurring through a period-doubling cascade, which he observed on the basis of Poincar'e maps. Thompson and co-workers offered a new perspective on the ship-capsize problem by considering the capsize process as dynamically equivalent to the escape of a ball rolling in a potential well, which is an intrinsically transient phenomenon. One of the first major contributions was the proposal of a diagram for the practical assessment of a hull's capsizability, which was known as the transient capsize diagram. Extensive cell-mapping and continuation studies were performed for a roll equation with direct or parametric excitation $[10,15,16]$. In the late 1980 s/early 1990 s, capsizing due to transient rolling had become a hot topic. Nayfeh and Sanchez presented numerical safe basins for the roll motion in beams [8]. The simulation of nonlinear rolling motions is also studies by Sanchez and Nayfeh in longitudinal waves [11]. Another significant step forward was the use of Melnikov analysis for predicting capsizing wave slopes in beam regular waves [2]. By use of realistic restoring representations, like a fifth- or higher-order polynomial, is, however, problematic when the analytical route is followed [14]. Bikdash et al. 
examined the equivalence between quadratic and cubic damping nonlinearities from a Melnikov perspective [1]. Hsieh et al. [4] adapted the Melnikov approach for a random excitation using the concept of Wiggins [18, 19] for phase flux transport out of the safe basin. This approach was extended for a biased vessel by Jiang et al. [5]. The interfacing of nonlinear dynamics and stochastic excitation have also been tackled by others [3]. The papers of Senjanovi'c and Falzarano with their associates formed a mainstream in nonlinear-dynamics investigations of ship capsize under stochastic wave excitation. Senjanovi'c et al. followed a repetitive simulation procedure in order to produce a chart giving the probability of capsize in terms of ship-motion direction and speed [12, 13].

In this paper Melnikov function and phase space flux are applied to analyze highly nonlinear roll and capsize of ships in random seas. Several factors, including significant wave frequency, significant wave height, nonlinear righting arms and damper characteristic are taken into account in the analysis of their influence on the phase space flux. Consider a fish vessel of 30.7 long and $6.9 \mathrm{~m}$ wide, sailing in the ISSC wave spectrum and the Melnikov function in the time domain is computed. The relation between the phase space flux and Melnikov function is gained and the influence of significant wave height on phase space flux is also attained. It is shown that phase space flux is growing continually along with significant wave height and the safe basin of ships is decreasing rapidly. Therefore, the osculating relations between the phase space flux and ship capsize are revealed and valuable reference will be provided for the ship design and safe standard.

\section{PHYSICAL MODEL FOR SHIP ROLL-MOTION}

Assume that the dynamics of large amplitude rolling of ships in random beam seas can be modeled by the following equation of motion of single degree of freedom (SDOF) in terms of the relative roll angle $\phi$ :

$$
\begin{aligned}
& \left(I_{44}+A_{44}(\omega)\right) \ddot{\phi}+B_{44}(\omega) \dot{\phi}+B_{44 q}(\omega) \dot{\phi}^{3}+\Delta G Z \\
& =F_{\text {sea }}(\tau)
\end{aligned}
$$

where $I_{44}$ is the rotational moment of inertia about an assumed rolling center, $A_{44}$ is the added moment of inertia due to the ambient fluid, $B_{44 q}$ is the nonlinear damping coefficient, $\Delta$ is displacement of ship, $G Z$ is the nonlinear righting arm in ship rolling, $F_{\text {sea }}(\tau)$ is the external excitation resulting from the random beam seas, and the over dot denotes differentiation with respect to time $t$.

The nonlinear restoring arm can be approximated reasonably well by the following odd cubic polynomial of $\phi$,

$$
G Z(f)=C_{1} \phi-C_{3} \phi^{3}
$$

where $C_{1}$ and $C_{3}$ are linear and nonlinear coefficients of the restoring arm.

The roll excitation moment in random beam seas can be expressed in the form as [6]

$$
F_{\text {sea }}(\tau)=I \alpha_{0} \omega_{0}^{2} \frac{\sqrt{2 \widetilde{\omega}}}{g} \sum_{n=1}^{N}(n \widetilde{\omega})^{2} \sqrt{S(n \widetilde{\omega})} \cos \left(\omega_{n} t+\xi_{n}\right)
$$

where $I=I_{44}+A_{44}(\omega), \alpha_{0}$ is the effective wave slope coefficient and assumed to be constant, $\omega_{0}$ is the natural frequency of initial roll, $\widetilde{\omega}$ is the step of wave frequency, $S$ is the spectral density function and $\xi_{n}$ is the random phase angle in the range of 0 to $2 \pi$.

Substituting those above-mentioned rolling parameters into equation (1) and let $x=\phi$, it can be written in the non-dimensional form as

$$
\ddot{x}(t)+\varepsilon \delta_{1} \dot{x}(t)+\varepsilon \delta_{2} \dot{x}^{3}(t)+x(t)-\alpha x^{3}=\varepsilon f(t)
$$

where $\varepsilon$ is a parameter of small values, $\varepsilon \delta_{1}$ and $\varepsilon \delta_{2}$ denote the dimensionless linear and quadratic viscous damping coefficient, respectively, and $\alpha$ denotes the strength of the nonlinearity. The non-dimensional terms are defined as

$$
\begin{aligned}
& x=\phi ; t=\omega_{0} t ; \varepsilon \delta_{1}=\frac{B_{44}(\omega)}{\Delta C_{1}} \omega_{0} ; \\
& \varepsilon \delta_{2}=\frac{B_{44 q}(\omega)}{I_{44}+A_{44}(\omega)} \omega_{0} ; \alpha=\frac{C_{3}}{C_{1}} ; \Omega=\frac{\omega}{\omega_{0}} ; \\
& \varepsilon f(t)=\frac{F_{\text {sea }}}{\Delta C_{1}} ; \omega_{0}=\sqrt{\frac{\Delta C_{1}}{I_{44}+A_{44}(\omega)}}
\end{aligned}
$$

Simplifying equation (4) in a first-order form yields

$$
\left\{\begin{array}{c}
\dot{x}(t)=y(t) \\
\dot{y}(t)=-x(t)+\alpha x^{3}(t)+\varepsilon\left(-\delta_{1} y(t)-\delta_{2} y^{3}(t)+\varepsilon f(t)\right)
\end{array}\right.
$$

Equation (5) represents an integrable Hamiltonian system. For an unperturbed and un-damped system, equation (3) can be further simplified to

$$
\left\{\begin{array}{c}
\dot{x}(t)=y(t) \\
\dot{y}(t)=-x(t)+\alpha x^{3}(t)
\end{array}\right.
$$


A closed form solution of equation (6) can be obtained as

$$
\left\{\begin{array}{c}
x_{0}(t)= \pm \sqrt{\frac{1}{\alpha}} \tanh \left(\frac{t}{\sqrt{2}}\right) \\
y_{0}(t)= \pm \sqrt{\frac{1}{2 \alpha}} \operatorname{sech}^{2}\left(\frac{t}{\sqrt{2}}\right)= \pm\left(\sqrt{\frac{1}{2 \alpha}}-\sqrt{\frac{\alpha}{2}} x_{0}^{2}\right)
\end{array}\right.
$$

where "+" denotes the positive part of the heteroclinic orbits and "-" represents the negative part of the heteroclinic orbits. The heteroclinic orbits are the boundary between capsizing and safety, which constitutes an important part in the following study of the paper.

\section{MELNIKOV FUNCTION FOR RANDOM EXCITATION}

Considering the single DOF system described by the following equation

$$
\dot{x}=f(x)+\varepsilon g(x, t)
$$

The Melnikov function for equation (6) can be expressed as

$$
M\left(t_{0}\right)=\int_{-\infty}^{+\infty} f\left(x_{0}(t)\right) \wedge g\left(x_{0}(t), t+t_{0}\right) d t
$$

where $\wedge$ is the Possion symbol, defined as

$$
a=\left\{\begin{array}{l}
a_{1} \\
a_{2}
\end{array}\right\}, b=\left\{\begin{array}{l}
b_{1} \\
b_{2}
\end{array}\right\}, a \wedge b=\left[a_{1} b_{2}-a_{2} b_{1}\right] .
$$

The coordinate of saddle points is $( \pm \sqrt{1 / \alpha}, 0)$, therefore the area of safe basin enclosed by the heteroclinic orbits is

$$
\begin{aligned}
A_{0} & =2 \int_{-\infty}^{+\infty} y_{0}^{+}(t) d x_{0}(t)=2 \int_{-\sqrt{1 / \alpha}}^{\sqrt{1 / \alpha}}\left(\sqrt{\frac{1}{2 \alpha}}-\sqrt{\frac{\alpha}{2}} x_{0}^{2}\right) d x_{0} \\
& =\frac{4 \sqrt{2}}{3 \alpha}
\end{aligned}
$$

Similarly, the Melnikov function for equation (5) can be derived as ,

$$
\begin{aligned}
M\left(t_{0}\right) & =\int_{-\infty}^{+\infty} y_{0}(t)\left(-\delta_{1} y_{0}(t)-\delta_{2} y_{0}^{3}(t)+f\left(t+t_{0}\right)\right) d t \\
& =\tilde{M}\left(t_{0}\right)-\bar{M}
\end{aligned}
$$

where $\bar{M}$ is the mean value of the melnikov function and is related to the damping of ship by

$$
\bar{M}=\int_{-\infty}^{+\infty} y_{0}(t)\left(\delta_{1} y_{0}(t)+\delta_{2} y_{0}^{3}(t) d t\right.
$$

$\tilde{M}\left(t_{0}\right)$ is the oscillatory part of the Melnikov function, and is related to the external excitation by

$$
\tilde{M}\left(t_{0}\right)=\int_{-\infty}^{+\infty} y_{0}(t) f\left(t+t_{0}\right) d t
$$

where

$$
f\left(t+t_{0}\right)=\frac{I \alpha_{0} \omega_{0}^{2} \pi}{C_{1} \Delta} \sum_{n=1}^{N} \frac{h_{n}}{\lambda_{n}} \cos \left[\Omega_{n}\left(t+t_{0}\right)+\xi_{n}\right]
$$

where $h_{n}, \lambda_{n}$ are wave height and wave length respectively, given by

$$
h_{n}=2 \sqrt{2 \widetilde{\omega} S(n \widetilde{\omega})}, \lambda_{n}=\frac{2 \pi g}{(n \widetilde{\omega})^{2}}, \Omega_{n}=\frac{\omega}{\omega_{0}}=\frac{n \widetilde{\omega}}{\omega_{0}}
$$

Substituting equation (7) and (14) into equation (13) yields

$$
\begin{aligned}
\tilde{M}\left(t_{0}\right) & =\frac{I \alpha_{0} \omega_{0}^{2} \pi^{2}}{C_{1} \Delta} \sum_{n=1}^{N} \sqrt{\frac{2}{\alpha}} \frac{h_{n} \Omega_{n}}{\lambda_{n} \sinh \left(\pi \Omega_{n} / \sqrt{2}\right)} \\
& \times \cos \left(\Omega_{n} t_{0}+\xi_{n}\right)
\end{aligned}
$$

\section{CALCULATION OF RATE OF PHASE SPACE FLUX}

The rate of phase space flux denotes the area of safe basin (The safe basin is chosen to be a region where displacements stay within some prescribed limits, that is , the safe basin is the region $\ell=\left\{(y, \dot{y}) \in \mathfrak{R}^{2}:|y|<b\right\}$ for a given $b>0$ ) that transports beyond the heteroclinic orbits and runs into the unbounded region during one period of external excitation. The larger the rate of phase space flux, the smaller the area of safe basin; therefore, its amplitude determines whether the ship's capsize or not.

If the external excitation is assumed to be a narrow band wave and the power spectral function of external excitation is $S_{f}(\Omega)(-\infty<\Omega<\infty)$, then the corresponding single-sided power spectral function is

$$
S_{f}^{+}=2 S_{f}(\Omega), \Omega \geq 0
$$

Now, the mean value of $\tilde{M}\left(t_{0}\right)$ is

$$
\begin{aligned}
E\left[\tilde{M}\left(t_{0}\right)\right] & =E\left[\int_{-\infty}^{+\infty} y_{0}(t) f\left(t+t_{0}\right) d t\right] \\
& =\int_{-\infty}^{+\infty} y_{0}(t) E\left[f\left(t+t_{0}\right)\right] d t
\end{aligned}
$$

Owing to $E[f(t)]=0$ therefore $E\left[\tilde{M}\left(t_{0}\right)\right]=0$. Then 
the mean value of Melnikov function is represented by the value of $-\bar{M}$.

In order to compute the mean square value of $\tilde{M}\left(t_{0}\right)$, the auto-correlation function of $\tilde{M}\left(t_{0}\right)$ must be defined firstly, i.e.,

$$
\begin{aligned}
R_{\tilde{M}}(\tau) & =\lim _{T \rightarrow \infty} \frac{1}{T} \int_{-\frac{1}{2} T}^{\frac{1}{2} T} \tilde{M}\left(t_{0}\right) \tilde{M}\left(t_{0}+\tau\right) d t \\
& =\int_{-\infty}^{+\infty} S_{\widetilde{M}}(\Omega) e^{-j \Omega \tau} d \Omega
\end{aligned}
$$

The power spectral density function of $\tilde{M}\left(t_{0}\right)$ can be expressed by

$$
S_{\tilde{M}}=2 \pi \tilde{M}^{*}(\Omega) \overline{\tilde{M}^{*}(\Omega)}=\frac{1}{2 \pi} \int_{-\infty}^{+\infty} R_{\tilde{M}}(\tau) e^{-j \Omega \tau} d \tau
$$

The over bar is used to denote the complex conjugate, and $\tilde{M}^{*}(\Omega)$ is the Fourier transform of $\tilde{M}\left(t_{0}\right)$, i.e.,

$$
\tilde{M}^{*}(\Omega)=\frac{1}{2 \pi} \int_{-\infty}^{+\infty} \tilde{M}\left(t_{0}\right) e^{-j \Omega t_{0}} d t_{0}
$$

From the random process theory we know that $R(\tau)$ is an even function, therefore $S_{\widetilde{M}}(\Omega)=S_{\tilde{M}}(-\Omega)$.

$$
E\left[\tilde{M}^{2}(t)\right]=\lim _{T \rightarrow \infty} \frac{1}{T} \int_{-\frac{1}{2} T}^{\frac{1}{2} T} \tilde{M}^{2}(t) d t=R_{\tilde{M}}=\int_{0}^{+\infty} S_{\tilde{M}}^{+}(\Omega) d \Omega
$$

where $S_{\widetilde{M}}^{+}=\left\{\begin{array}{ll}2 S_{\tilde{M}} & \Omega>0 \\ 0 & \Omega \leq 0\end{array}\right.$.

Considering the Fourier transform of $\tilde{M}\left(t_{0}\right)$, which can be expressed in terms of the Fourier transforms of the input $f(t)$ and the velocity along the heteroclinic solution $y_{0}(t)$ as

$$
\begin{aligned}
\tilde{M}^{*}(\Omega) & =\frac{1}{2 \pi} \int_{-\infty}^{+\infty}\left(\int_{-\infty}^{+\infty} y_{0}(t) f\left(t+t_{0}\right) d t\right) e^{-j \Omega t_{0}} d t_{0} \\
& =\frac{1}{2 \pi} \int_{-\infty}^{+\infty} y_{0}(t)\left(\int_{-\infty}^{+\infty} f\left(t+t_{0}\right) e^{-j \Omega t_{0}} d t_{0}\right) d t
\end{aligned}
$$

Equation (14) can be simplified by scaling time according to $t+t_{0}=\xi$, and it leads to

$$
\begin{aligned}
\tilde{M}^{*}(\Omega) & =\frac{1}{2 \pi} \int_{-\infty}^{+\infty} y_{0}(t) \int_{-\infty}^{+\infty} f(\xi) e^{-j \Omega \xi t} d \xi e^{-j \Omega t} d t \\
& =2 \pi f^{*}(\Omega) \overline{y_{0}^{*}(\Omega)}
\end{aligned}
$$

where the over line denotes the complex conjugate.

Using equation (23) and the random vibration theory, it follows that

$$
\begin{aligned}
S_{\tilde{M}}(\Omega) & =2 \pi \tilde{M}^{*}(\Omega) \overline{\tilde{M}^{*}(\Omega)} \\
& =2 \pi\left(2 \pi f^{*}(\Omega) \overline{y_{0}^{*}(\Omega)}\right)\left(2 \pi \overline{f^{*}(\Omega)} y_{0}^{*}(\Omega)\right) \\
& =2 \pi S_{y_{0}}(\Omega) S_{f}(\Omega)
\end{aligned}
$$

Therefore the single sided power spectral of $\tilde{M}\left(t_{0}\right)$ can be written as

$$
\begin{aligned}
S_{\widetilde{M}}^{+} & =2 S_{\tilde{M}}(\Omega)=2\left[2 \pi S_{y_{0}}(\Omega) S_{f}(\Omega)\right] \\
& =2 \pi S_{y_{0}}(\Omega) S_{f}^{+}(\Omega)
\end{aligned}
$$

The mean square value of $\tilde{M}\left(t_{0}\right)$ is obtained as

$$
\begin{aligned}
\sigma_{M\left(t_{0}\right)}^{2} & =\sigma^{2} \tilde{M}\left(t_{0}\right) \\
& =E\left[\tilde{M}\left(t_{0}\right)-E \tilde{M}\left(t_{0}\right)\right]^{2}=\int_{-\infty}^{+\infty} S_{\tilde{M}}(\Omega) d \Omega \\
& =\int_{0}^{+\infty} 2 \pi S_{y_{0}}(\Omega) S_{f}^{+}(\Omega) d \Omega
\end{aligned}
$$

Considering $y_{0}=\sqrt{\frac{1}{2 \alpha}} \operatorname{sech}^{2}\left(\frac{t}{\sqrt{2}}\right)$ the power spectral of the roll velocity along the heteroclinic is given by

$$
S_{y_{0}}=\frac{\pi}{\alpha}\left(\frac{\Omega}{\sin h(\Omega \pi / \sqrt{2})}\right)^{2}
$$

The average rate of phase flux for the case of random excitation during one period of excitation $T$ is given by

$$
\Phi=\lim _{T \rightarrow \infty} \frac{\varepsilon}{2 T} \int_{-T}^{T} M^{+}(\xi) d \xi=\lim _{T \rightarrow \infty} \frac{\varepsilon}{2 T} \int_{-T}^{T}(\tilde{M}(\xi)-\bar{M})^{+} d \xi
$$

where $g^{+}(\xi)= \begin{cases}g(\xi) & g(\xi)>0 \\ 0 & g(\xi) \leq 0\end{cases}$

Based on the previous analysis, $M\left(t_{0}\right)$ is a random process with Gaussian distribution with respect to $t_{0}$, so the expression for $\Phi$ can be attainted by carrying the integral,

$$
\Phi(\sigma, \bar{M})=\varepsilon E\left[\left(\tilde{M}_{q}(\xi)-\bar{M}\right)^{+}\right]=\varepsilon \int_{\bar{M}}^{+\infty}(z-\bar{M}) p(z) d z
$$

$$
\begin{aligned}
& \text { Let } \frac{z}{\sigma}=x, d z=\sigma d x \\
& \begin{aligned}
\Phi(\sigma, \bar{M}) & =\varepsilon \sigma \int_{\frac{M}{\sigma}}^{+\infty}\left(\frac{1}{\sqrt{2 \pi}}\right)\left(x-\frac{\bar{M}}{\sigma}\right) \exp \left(-\frac{x^{2}}{2}\right) d x \\
& =\varepsilon\left[\sigma p\left(\frac{\bar{M}}{\bar{\sigma}}\right)-\bar{M}+\bar{M} P\left(\frac{\bar{M}}{\bar{\sigma}}\right)\right]
\end{aligned}
\end{aligned}
$$


Table 1. Main parameters of fishing vessel

\begin{tabular}{cccc}
\hline Length overall $\left(\mathrm{L}_{\mathrm{oa}}\right)$ & $30.70 \mathrm{~m}$ & $I_{44}+A_{44}$ & $1.078 \times 10^{6} \mathrm{~kg}-\mathrm{m}^{2}$ \\
\hline Length between perpendiculars $\left(\mathrm{L}_{\mathrm{pp}}\right)$ & $25.00 \mathrm{~m}$ & Nature frequency $\left(\omega_{0}\right)$ & $1.32 \mathrm{rad} / \mathrm{sec}$ \\
Breadth $(\mathrm{B})$ & $6.90 \mathrm{~m}$ & Linear damping coefficient $\left(B_{44}\right)$ & $2.242 \times 10^{4} \mathrm{~kg}-\mathrm{m}^{2} / \mathrm{s}$ \\
Height $(\mathrm{H})$ & $4.96 \mathrm{~m}$ & Nonlinear damping coefficient $\left(B_{44 q}\right)$ & $1.777 \times 10^{4} \mathrm{~kg}-\mathrm{m}^{2}$ \\
Draught $(\mathrm{T})$ & $2.67 \mathrm{~m}$ & Linear restoring coefficient $\left(C_{1}\right)$ & $0.962 \mathrm{~m}$ \\
Displacement $(\Delta)$ & $195 \mathrm{t}$ & Nonlinear restoring coefficient $\left(C_{3}\right)$ & $0.218 \mathrm{~m}$ \\
\hline
\end{tabular}

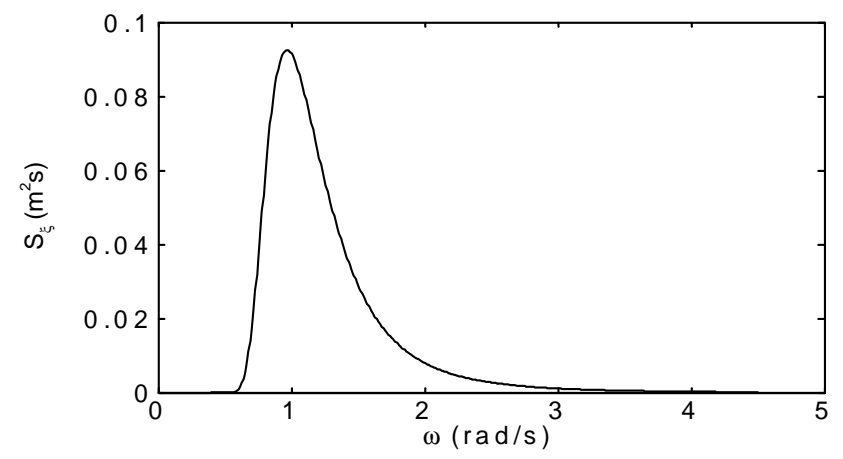

Fig. 1. Wave power spectrum.

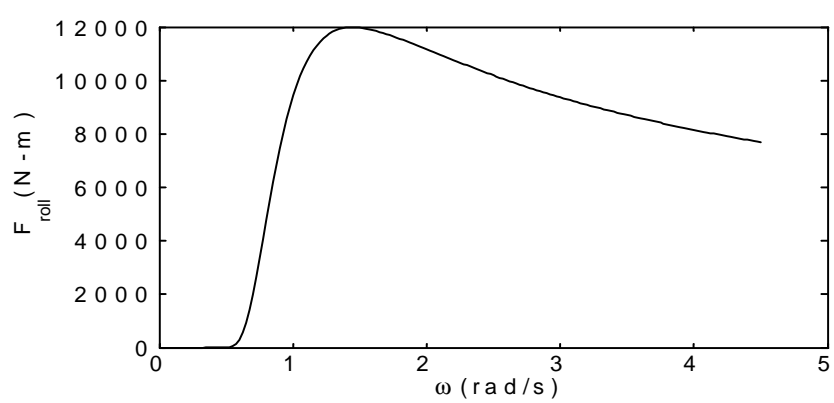

Fig. 2. Roll moment amplitude.

where

$$
p(x)=\frac{1}{\sqrt{2 \pi}} \exp \left(-\frac{x^{2}}{2}\right) ; P(x)=\int_{-\infty}^{x} p(x) d \xi
$$

\section{NUMERICAL RESULTS}

For illustration of rolling motion, a fishing vessel with the following parameters is chosen as an example.

The wave spectrum used in this paper is the ISSC two-parameter spectral formula,

$$
S^{+}\left(\Omega, H_{s}\right)=0.11 H_{s}^{2} \frac{\omega_{z}^{4}}{\Omega^{5} \omega_{0}^{5}} \exp \left[-0.44\left(\frac{\omega_{z}}{\Omega \omega_{0}}\right)^{4}\right]
$$

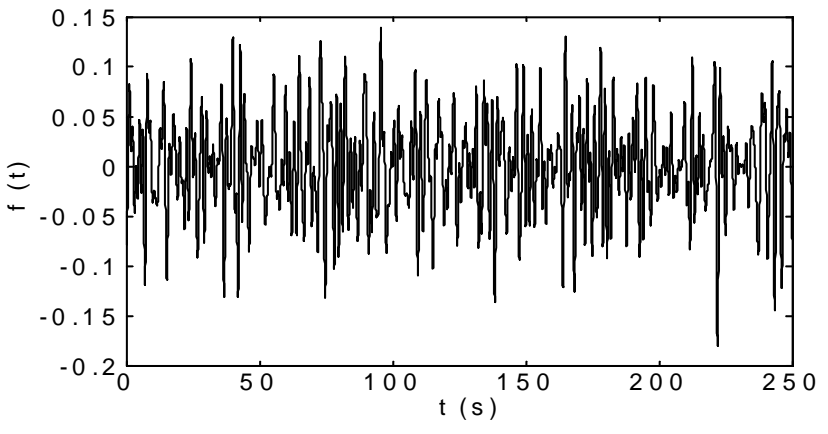

Fig. 3. Excitation $f(t)$.

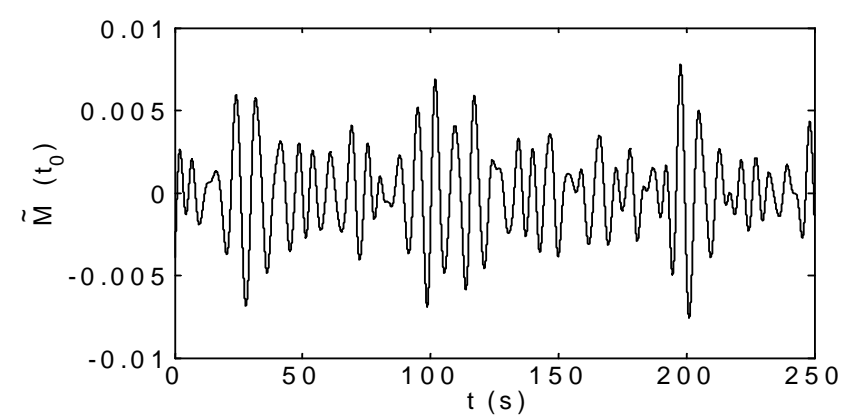

Fig. 4. The oscillation part of melnikov function.

where $\omega_{0}$ is the natural frequency of the ship, $\omega_{z}$ and $H_{s}$ are the significant wave frequency and height respectively, $\Omega=\frac{\omega}{\omega_{0}}$.

The generation of random wave excitation of rolling motion is illustrated for the case of significant wave height $H_{s}=1$ mand significant wave periodic $T_{s}=5 \mathrm{~m}$. The wave power spectrum up to frequency $\omega=4.5 \mathrm{rad} /$ $\mathrm{s}$ is taken into account. The frequency step is chosen to be $\widetilde{\omega}=0.025 \mathrm{rad} / \mathrm{s}$. Thus, the total number of harmonic waves forming a random wave pattern is $N=\frac{\omega}{\widetilde{\omega}}=180$. The effective wave slope coefficient is $\alpha_{0}=0.729$.

The analysis results are shown in Figures 1 to 8 . While Figure 1 shows the wave power spectrum and the roll moment amplitude per unit significant wave height 


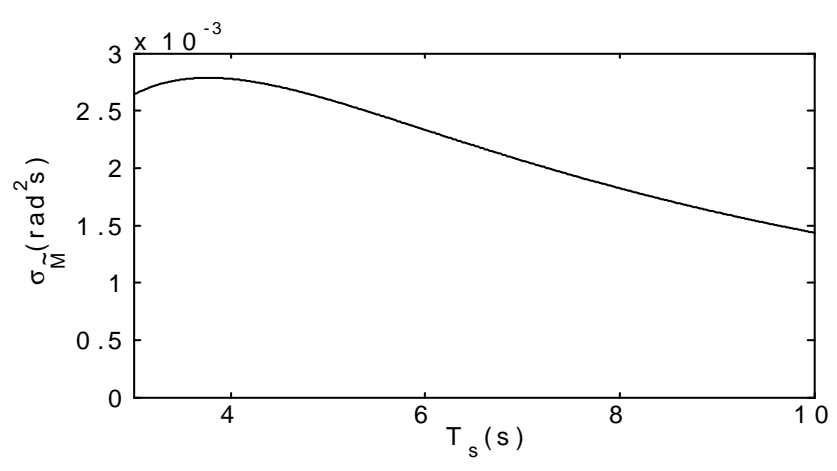

Fig. 5. Root mean square value of $\tilde{M}\left(t_{0}\right)$.

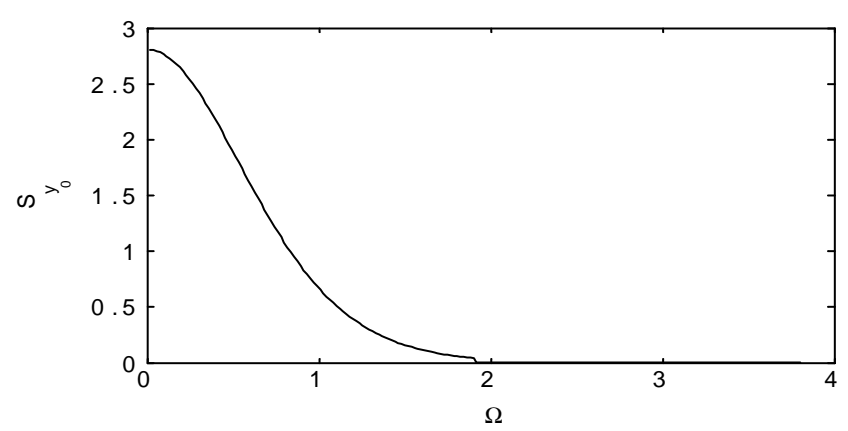

Fig. 6. Transfer function versus $\Omega$.

is shown in Figure 2, in which $F_{\text {roll }}(\omega)$ is resulted from the pressure due to the waves with small slopes acting on a fixed vessel in the upright position.

Figure 3 and Figure 4 show the typical time traces of the excitation and a corresponding Melnikov function, respectively.

Figure 5 shows the mean square values for $\tilde{M}\left(t_{0}\right)$ by the finite-frequency approximation over a range of characteristic wave periods. Figure 6 shows the power spectrum of the roll velocity along the heteroclinic orbits.

Figure 7 shows the rate of the phase space flux with respect to $\bar{M}$ ( $\bar{M}$ is a function of ship's form). The rate of the phase space flux along with significant wave period is shown in Figure 8. From this figure it can be seen that the rate of the phase space flux $\Phi(\sigma, \bar{M})>0$ when $H_{s}=5.6 \mathrm{~m}$. When the wave height reach certain value, the rate of phase space flux is rising linearly with $H_{s}$ and the area of the ship's safe basin is decreasing dramatically and it leads the ship to capsize finally.

\section{CONCLUSIONS}

The approach of utilizing Melnikov function and the phase space flux to determine the safety of ships in random beam seas are discussed in this paper. Ship

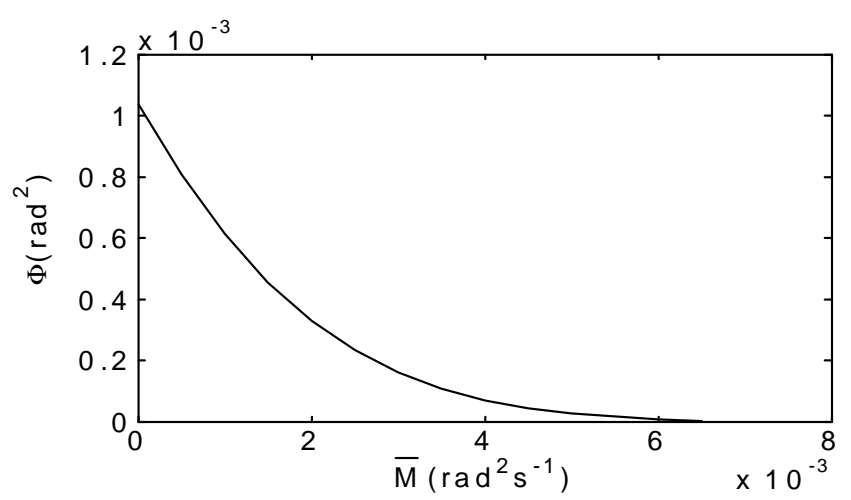

Fig. 7. Phase space flux versus to the mean part of melnikov function.

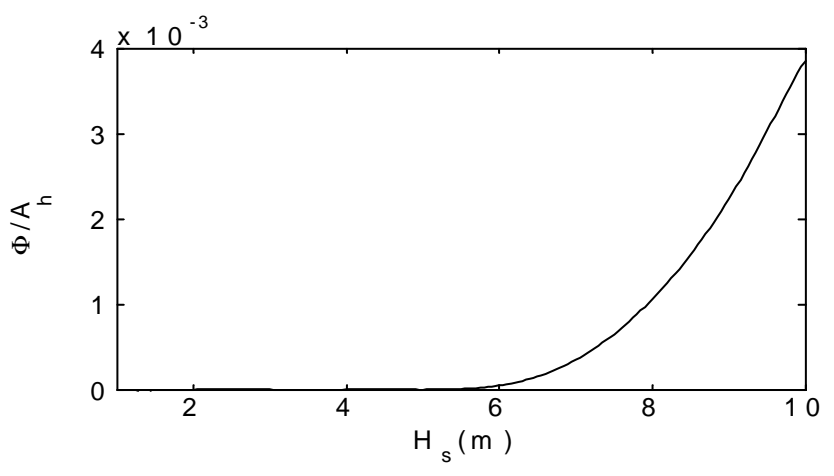

Fig. 8. Variation of $\Phi / A_{h}$ as a function of $H_{s}$.

rolling motion is simulated by a single degree freedom system. Nonlinear vicious damping and the restoring moment presented by a high order polynomial are taken into account. Random wave excitation has found to be depending on the relevant wave slope energy spectrum for a given sea state and encounter frequency. Numerical analysis results have presented with the following some conclusions:

(1) The mean value of Melnikov function $\bar{M}$ has a prodigious influence on the rate of the phase space flux, the larger the $\bar{M}$, and the less the $\Phi$. Therefore reasonably selecting the form of a ship will directly affect the safety of the ship.

(2) When the significant period of waves is far away from the initial roll natural period, the influence on the phase space flux is little, while the significant wave height plays an important role on the rate of phase space flux. When the wave height reaches certain value, the rate of phase space flux is rising linear with Hs and the area of the ship's safe basin is decreasing dramatically.

(3) In the study of this paper, it can be found that 
the wave power spectrum, the step of wave frequency and the random phase angle affect the amplitude of roll moment, the amplitude of roll moment and the oscillatory part of Melnikov function remarkably. Therefore reasonably choosing the step of wave frequency and the random phase angle are quite important.

\section{REFERENCES}

1. Bikdash, M., Balachandran, B., and Nayfeh, A., "Melnikov Analysis for a Ship with a General Rolldamping Model," Nonlinear Dyn., Vol. 6, pp. 101-124 (1994).

2. Falzarano, J.M., Shaw, S.W., and Troesch, A.W., “Application of Global Methods for Analyzing Dynamical Systems to Ship Rolling and Capsizing," Int. J. Bifurcat. Chaos, Vol. 2, pp. 101-116 (1992).

3. Francescutto, A., "On the Probability of Large-amplitude Rolling and Capsizing as a Consequence of Bifurcations," Proc. Symp. Dynamics of Marine Vehicles and Structures in Waves, London, pp. 291-304 (1990).

4. Hsieh, S., Troesch, A.W., and Shaw, S.W., "A Nonlinear Probabilistic Method for Predicting Vessel Capsizing in Random Beam Seas," Proc. R. Soc. Lond., A 446, pp. 195-211 (1994).

5. Jiang, C., Troesch, A.W., and Shaw, S.W., "Highly Nonlinear Rolling Motion of Biased Ships in Random Beam Seas," J. Ship Res., Vol. 40, pp. 125-135 (1996).

6. Nayfeh, A.H. and Khdeir, A.A., "Nonlinear Rolling of Ships in Regular Beam Seas," Int. Shipbuild. Prog., Vol. 33, pp. 40-49 (1986).

7. Nayfeh, A.H. and Khdeir, A.A., "Nonlinear Rolling of Biased Ships in Regular Beam Seas," Int. Shipbuild. Prog., Vol. 33, pp. 89-93 (1986).

8. Nayfeh, A.H. and Sanchez, N.E., "Stability and Complicated Rolling Responses of Ships Irregular Beam Seas," Int. Shipbuild. Prog., Vol. 37, pp. 331-352 (1990).
9. Papanikolaou, A. and Zaraphonitis, G., "Computer-aided Simulations of Large Amplitude Roll Motions of Ships in Waves and of Dynamic Stability," Int. Shipbuild. Prog., Vol. 34, pp. 198-206 (1987).

10. Rainey, R.C.T. and Thompson, J.M.T., "Transient Capsize Diagram New Method of Quantifying Stability in Waves," J. Ship Res., Vol. 35, pp. 58-62 (1991).

11. Sanchez, N.E. and Nayfeh, A.H., "Nonlinear Rolling Motions of Ships in Longitudinal Waves," Int. Shipbuild. Prog., Vol. 37, pp. 247-272 (1990).

12. Senjanovic, I., Cipric, G., and Pavunov. J., "Nonlinear Ship Rolling and Capsizing in Rough Sea," Brodogradnja, Vol. 44, No. 1, pp. 19-24.

13. Senjanovic, I. and Fan, Y., "Numerical Simulation of a Ship Capsizing in Irregular Waves," Chaos Solitons Fractals, Vol. 5, No. 5, pp. 727-737 (1995).

14. Scolan, Y.M., "Technical Note on Ship Rolling Associated to High Degree Polynomial Restoring Moment by Using the Melnikov Method," Appl. Ocean Res., Vol. 19, pp. 225-234 (1998).

15. Thompson, J.M.T. and Soliman, M.S., "Fractal Control Boundaries of Driven Oscillators and Their Relevance to Safe Engineering Design," Proc. R. Soc. Lond., A 428, pp. 1-13 (1990).

16. Thompson, J.M.T., Rainey, R.C.T., and Soliman, M.S., "Mechanics of Ship Capsize under Direct and Parametric Wave Excitation," Phil. Trans. R. Soc. Lond., A 428, pp. 1-13 (1992).

17. Virgin, L.N., "The Nonlinear Rolling Response of a Vessel Including Chaotic Motions Leading to Capsize in Regular Seas," Appl. Ocean Res., Vol. 9, pp. 89-95 (1987).

18. Wiggins, S., Chaotic Transport in Dynamical Systems, Springer-Verlag, New York (1992).

19. Wiggins, S., Introduction to Applied Nonlinear Dynamical Systems and Chaos, Heidelber, New York (1990). 